\title{
BRIEF REPORT \\ Probing affective pictures: Attended startle and tone probes
}

\author{
BRUCE N. CUTHBERT, HARALD T. SCHUPP, MARGARET BRADLEY, \\ MARK MCMANIS, AND PETER J. LANG \\ Department of Clinical and Health Psychology, University of Florida, Gainesville, USA
}

\begin{abstract}
Reflexive eyeblinks to a startle probe vary with the pleasantness of affective pictures, whereas the corresponding P300 varies with emotional arousal. The impact of attention to the probe on these effects was examined by varying task and probe type. Probes were either nonstartling tones or startling noises presented during affective picture viewing. Half the participants performed a task requiring attention to the probes; the other participants were told to ignore the probes. Blinks to the startle probe varied with picture pleasantness for both task and nontask conditions. In contrast, P300 magnitudes for both startle and tone probes were reduced during emotionally arousing pictures, irrespective of pleasantness, in task and nontask conditions. Further, attending to the startle probe prompted an augmentation of N100 during unpleasant pictures. The data suggest that affective modulation of probe responses reflects obligatory processes in picture perception.
\end{abstract}

Descriptors: Event-related potentials, P300, Startle, Emotion, Attention

In a recent study, two concurrent measures of the evoked startle response, the elicited blink reflex and the event-related potential (ERP), were recorded while participants viewed emotionally evocative pictures (Schupp, Cuthbert, Bradley, Birbaumer, \& Lang, 1997). Consistent with earlier studies, the reflexive blink response was modulated by picture valence, that is, largest for unpleasant and smallest for pleasant pictures (for an overview, see Lang, Bradley, \& Cuthbert, 1990). The magnitude of the elicited P300 response to the startle probe was also modulated by picture content but with a different valence pattern: P300 responses were reduced similarly for both types of affective pictures (pleasant and unpleasant) compared with neutral pictures.

One explanation of the ERP result is that perceptual processing of emotionally intense pictures requires more attentional resources. Thus, fewer resources are available for processing the probe stimulus, and the reduction in probe attention is reflected in the diminished P300. This interpretation suggests that the attentional and emotional properties of the affective pictures could be explored

This work was supported in part by National Institute of Mental Health grants MH37757, MH41950, MH43975, and MH52384 and National Institute of Aging grant AG09779 to P. J. Lang, and by a grant from the Deutsche Forschungsgemeinschaft (Schu 1074/2-1) to H. T. Schupp.

These data were presented in part as a poster at the 36th convention of the Society for Psychophysiological Research in Vancouver, Canada (Cuthbert, Schupp, Bradley, McManis, \& Lang, 1996).

Harald T. Schupp's present address: University of Greifswald, Germany. Mark McManis's present address: Harvard University, Boston, MA, USA.

Address requests for reprints to: Bruce Cuthbert, NIMH Center for the Study of Emotion and Attention (CSEA), Box 100165 HSC, Gainesville, FL 32610-0165, USA. E-mail: brucec@nervm.nerdc.ufl.edu. using various probe paradigms. However, in most studies of affective startle modulation, participants were instructed to ignore the blink-eliciting probe. In contrast, a large literature has documented that responses to startle probes can be reliably modulated by probe-relevant attentional manipulations. For instance, the eyeblink reflex response is reliably augmented when attention is directed to the modality in which the startle probe is presented and inhibited when the participant attends to a modality that mismatches that of the probe (e.g., Anthony \& Graham, 1985; Hackley \& Graham, 1987).

No data exist as to whether such attentional effects may influence affective modulation. Accordingly, a primary goal of the current study was to examine the effects of task-related attention to the probe upon the eyeblink and ERP responses to the startle probe. For one group of participants, the probe provided information relevant to a postpicture task, and a second group received the usual "ignore-the-probe" instructions. To the extent that emotional responding is automatic and obligatory, affect modulation should be independent of the probe's signal value, so both groups would be expected to show affective modulation of startle and ERP responses.

A second goal of the experiment was to test whether the pattern of P300 modulation across picture contents is unique to the startle probe. According to Graham (1992), startle has special status as a processing interrupt, and Lang et al. (1990) viewed startle as a defense unconditioned stimulus (UCS) that is specifically primed by emotional foregrounds. Both views suggest that the startle P300, like the blink reflex, could be modulated differently from responses to less compelling probe stimuli. To test this hypothesis, low-intensity tones were included as a second probe type. When presented with a slow rise time, tones do not produce reliable 
startle reflexes. In contrast, picture encoding demands are the same regardless of probe characteristics. Thus, a tone ERP measured during emotionally arousing pictures may still reflect greater attention to the picture foreground and include a reduced P300.

A further consideration is the influence of emotional pictures on the sensory ERP, in particular N100. This negative wave, occurring around $100 \mathrm{~ms}$ poststimulus, has been shown to be enhanced when the participant is directed to attend to the eliciting stimuli. The N100 is also sensitive to arousal and the physical intensity of stimuli (Näätänen \& Picton, 1987). In the present study, we assessed the extent to which the probe N100 may also be modulated by an intense emotional picture, in interaction with attention toward the probe and the startle properties of the probe stimulus.

\section{Method \\ Participants}

Forty volunteers with normal hearing (20 women, 20 men) from the University of Florida introductory psychology course participated as part of a class requirement.

\section{Materials}

Fifty-four color pictures were chosen from the International Affective Picture System (IAPS; Center for the Study of Emotion and Attention, 1996), depicting 18 unpleasant events (e.g., snakes, mutilations), 18 pleasant events (e.g., sports, opposite sex nudes), and 18 neutral events (e.g., neutral faces, household objects). These were the same pictures as used in Schupp et al. (1997).

The acoustic startle stimulus consisted of a 50-ms, 95-dB (A) burst of white noise with instantaneous rise time. The tone stimulus was $800 \mathrm{~Hz}, 65-\mathrm{dB}$ (A), 100-ms duration, and 50-ms rise time. Startle and tone probes were presented binaurally through matched Telephonics stereo headphones. In both a 6-s picture and a 6-s postpicture period, either a startle or tone stimulus $(50 / 50$ probability) was delivered at a random time point $2.5-5 \mathrm{~s}$ after the beginning of the period. Nine startles and nine tones occurred at each of the three valence levels of the picture period and again during the postpicture period.

\section{Procedure}

After attachment of electrodes, the participants were familiarized with the affective rating procedure. They were told that a series of slides would be presented (6 s) and that each picture should be viewed the entire time it was on the screen. One group of participants $(n=20)$ was told to ignore the startle and tone stimuli, and the other group performed a choice reaction time task at the second (postpicture) probe, pressing a button with the middle or index finger to indicate whether the probe was the same as (e.g., startlestartle) or different from (e.g., startle-tone) the picture probe. A variable interval (12-18 s) occurred between each trial. Two neutral pictures served as practice trials.

\section{Data Collection and Reduction}

The electroencephalogram (EEG) and vertical and horizontal electrooculogram (VEOG and HEOG) were amplified and bandpass filtered from 0.1 to $35 \mathrm{~Hz}$. Data were collected at $125 \mathrm{~Hz}$ from $1 \mathrm{~s}$ before until $2 \mathrm{~s}$ after probe onset. EEG was recorded from $\mathrm{Fz}, \mathrm{Cz}$, and $\mathrm{Pz}$ and converted offline to a linked mastoid reference. $(\mathrm{Cz}$ was used as the reference for all other sites, including separate mastoid derivations, during recording.) All individual trials with EEG activity above $\pm 150 \mu \mathrm{V}$ were excluded from further analy- sis, and the EEG was then corrected for eye movement and blink artifacts (Miller, Gratton, \& Yee, 1988). ${ }^{1}$ The N100 base-to-peak of the ERP was derived as the maximum negative deflection within a window of 64-192 ms; the P300 component was scored as a base-to-peak amplitude within a window beginning at N2 latency and continuing until $504 \mathrm{~ms}$ (for additional details, see Schupp et al., 1997). The eyeblink component of the startle reflex was measured as the base-to-peak deflection of the VEOG in the same window as the N100.

\section{Data Analysis}

The startle eyeblink was analyzed in a 2 (task: attended, unattended) $\times 3$ (affect: pleasant, neutral, unpleasant) mixed-design analysis of variance (ANOVA). N100 and P300 analyses included the additional variables of probe type (startle, tone) and location $(\mathrm{Fz}, \mathrm{Cz}, \mathrm{Pz})$. Effects involving repeated measures were evaluated using a multivariate test statistic (Wilks's lambda), with Bonferroniadjusted degrees of freedom used for pairwise comparisons of the three affective categories. ERPs in the postpicture period and reaction times did not differ by valence condition, and these data are not reported here.

\section{Results}

\section{Startle Blink Magnitude ${ }^{2}$}

Eyeblink magnitude was significantly modulated by picture pleasantness, affect $F(2,37)=3.6, p<.05$. Potentiated startle reflexes occurred when the probe was presented during unpleasant rather than during pleasant pictures, $F(1,39)=7.4, p<.01$ (Table 1). More critically, attention to the startle probe did not change these modulatory effects, Affect $\times$ Task $F(2,37)<1$.

\section{The P300 Wave}

Figure 1 illustrates the ERP waveforms as a function of affect, probe type, and probe task, and Table 1 lists the component scores used in the analyses. Replicating our earlier result, the P300 wave was significantly modulated by picture pleasantness, affect $F(2,37)=6.7, p<.01$. P300 amplitudes were reduced when viewing pleasant and unpleasant pictures as opposed to neutral images, $F(1,39)=13.2$ and $9.1, p \mathrm{~s}<.01$, respectively, whereas P300 amplitudes to probes during pleasant and unpleasant pictures were not different, $F(1,39)=1.0, n s$. Consistent with the blink results, the same pattern of affective modulation was obtained regardless of whether the probes were attended or ignored, Affect $\times$ Task $F(2,37)<1$, whereas an overall effect of task was not observed, $F<1$. The pattern of affective modulation also did not differ as a function of whether the probe was a startling stimulus or a tone, Affect $\times$ Probe Type $F(2,37)=2.1, n s$, indicating that P300 modulation by picture content occurs for both types of secondary probe stimuli. To document this effect more thoroughly, separate analyses showed reliable effects for both startle and tone probes, $F_{\mathrm{S}}(2,38)=6.4, p<.005$, and 3.6, $p<0.04$, respectively. Startle probes resulted in larger overall P300 amplitudes than tones, $F(2,37)=46.8, p<.001$.

\footnotetext{
${ }^{1}$ Nine trials were averaged for each Probe Type $\times$ Valence condition, a number that has proved sufficient in prior research due to the extended time intervals between probes. A total of 31 trials were rejected out of 2,160 total trials across all participants, leading to 7 trials for three cell averages, and 8 trials in the other affected cells.

2 The analysis of the m. orbicularis oculi revealed similar modulation of the blink reflex.
} 
Table 1. N1 and P3 Amplitudes ( $\mu$ V) for Attended and Unattended Startle and Tone Probes, and Blink Responses (vertical EOG, $\mu v$ ) for Startle Probes

\begin{tabular}{|c|c|c|c|c|c|}
\hline \multirow[b]{2}{*}{ Site } & \multirow[b]{2}{*}{ Affect } & \multicolumn{2}{|c|}{ Startle probe } & \multicolumn{2}{|c|}{ Tone probe } \\
\hline & & Task & No task & Task & No task \\
\hline \multicolumn{6}{|c|}{ N1 amplitude } \\
\hline \multirow[t]{3}{*}{$\mathrm{Fz}$} & Pleasant & 14.2 & 15.8 & 9.8 & 7.7 \\
\hline & Neutral & 15.1 & 15.5 & 10.0 & 7.1 \\
\hline & Unpleasant & 17.7 & 15.6 & 8.5 & 6.8 \\
\hline \multirow[t]{3}{*}{$\mathrm{Cz}$} & Pleasant & 22.9 & 22.5 & 11.0 & 8.6 \\
\hline & Neutral & 25.3 & 23.9 & 11.7 & 8.3 \\
\hline & Unpleasant & 28.0 & 24.7 & 9.8 & 8.9 \\
\hline \multirow[t]{3}{*}{$\mathrm{Pz}$} & Pleasant & 8.8 & 10.4 & 7.5 & 6.7 \\
\hline & Neutral & 10.6 & 12.1 & 8.3 & 7.3 \\
\hline & Unpleasant & 12.7 & 11.3 & 6.1 & 8.2 \\
\hline \multicolumn{6}{|c|}{ P3 amplitude } \\
\hline \multirow[t]{3}{*}{$\mathrm{Fz}$} & Pleasant & 12.1 & 12.4 & 6.4 & 5.7 \\
\hline & Neutral & 12.8 & 15.2 & 8.0 & 8.2 \\
\hline & Unpleasant & 12.8 & 14.3 & 7.7 & 7.1 \\
\hline \multirow[t]{3}{*}{$\mathrm{Cz}$} & Pleasant & 12.1 & 9.3 & 8.5 & 7.0 \\
\hline & Neutral & 17.1 & 17.1 & 10.6 & 9.3 \\
\hline & Unpleasant & 10.6 & 11.5 & 10.2 & 7.9 \\
\hline \multirow[t]{3}{*}{$\mathrm{Pz}$} & Pleasant & 19.2 & 15.4 & 10.9 & 8.0 \\
\hline & Neutral & 22.0 & 19.1 & 11.9 & 9.9 \\
\hline & Unpleasant & 17.3 & 16.4 & 11.8 & 6.7 \\
\hline \multicolumn{6}{|c|}{ Vertical EOG (startle only) } \\
\hline & Pleasant & 105.9 & 102.1 & & \\
\hline & Neutral & 111.8 & 109.9 & & \\
\hline & Unpleasant & 120.3 & 118.5 & & \\
\hline
\end{tabular}

The modulation of the P300 by picture pleasantness varied across electrode sites, Affect $\times$ Location $F(4,35)=4.4, p<.01$. At $\mathrm{Cz}$ and $\mathrm{Pz}, \mathrm{P} 300$ amplitudes were reduced for pleasant and unpleasant as opposed to neutral pictures, $F \mathrm{~s}(1,38)>7.1, p<$ .015. At Fz, only pleasant as opposed to neutral pictures resulted in reduced P300 amplitude, $F(1,38)=6.6, p<.015$.

\section{The N100 Wave}

The effect of picture pleasantness on the N100 peak varied as a function of task and probe type-Affect $\times$ Probe Type $F(2,37)=$ 7.7, $p<.01$; Affect $\times$ Probe Type $\times$ Task $F(2,37)=5.8, p<$ .01 -but not of task, $F(1,38)<1$. To assess affective modulation, separate analyses for tone and startle probes were conducted. For the startle probes, affective modulation of the N100 differed as a function of task, Affect $\times$ Task $F(2,37)=4.4, p<.02$. As shown in Figure 1 (left panel), participants attending to the probes had a larger N100 for unpleasant than for pleasant or neutral pictures, $F(1,19)=48.3$ and $8.3, p<.01$, respectively, affect $F(2,18)=$ $24.4, p<.001$. No affective modulation was observed when the startle probes were ignored (Figure 1, right panel). In contrast, the analysis of the tone probe N100s revealed no modulation as a simple function of picture pleasantness or as a function of affect and task, probe type, or location. As expected, startle probes elicited a much larger N100 than did tone probes, $F(1,38)=77.6$, $p<.001$.

\section{Control Analysis for Attended Startle Probes}

Because the peaks of both N100 and the eyeblink are very close in time, an analysis of the EEG uncorrected for ocular activity was
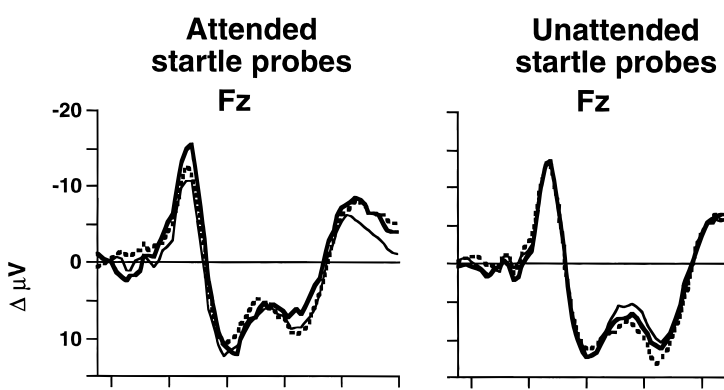

Fz
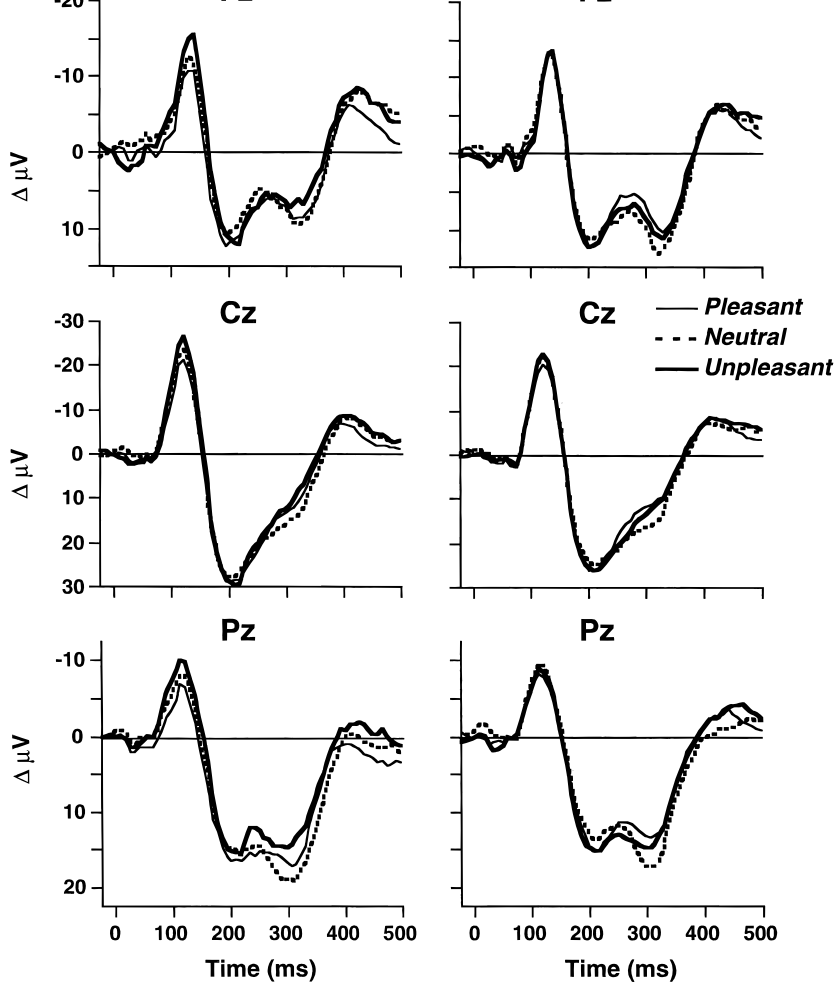

Figure 1. Startle probe synchronized grand average waveforms for $\mathrm{Fz}, \mathrm{Cz}$, and $\mathrm{Pz}$ during picture processing, separately for each valence category (pleasant, neutral, and unpleasant). Left: Waveforms for attended probes. Right: Waveforms for unattended startle probes.

performed to assess the possibility that the N100 effect was an artifact of the eye-movement correction procedure. Attended startle probes showed larger N100 responses for unpleasant than for pleasant pictures, although the main effect for affect only approached significance, $F(2,18)=2.8, p=.08$. Because the eyeblink distortion of the EEG is most prominent at $\mathrm{Fz}$ and less pronounced toward more posterior sites, single lead ANOVAs were conducted. Both $\mathrm{Cz}$ and $\mathrm{Pz}$ showed larger N100 for unpleasant $(18.3$ and $7.1 \mu \mathrm{V})$ than for pleasant pictures $(14.9$ and $4.0 \mu \mathrm{V})$, $F \mathrm{~s}(1,19)>7.6, p \mathrm{~s}<.015$. In contrast, $\mathrm{Fz}$ showed no significant effect.

\section{Discussion}

Consistent with many other studies, blink reflexes were potentiated when viewing unpleasant as opposed to pleasant pictures for participants who were instructed to ignore the startle probe. Moreover, an equally strong pattern of affective blink modulation was obtained whether or not the startle probes were explicitly attended. Thus, affective modulation of the startle blink is clearly not disrupted by simply directing attention to the probe. This conclusion received further support in the preliminary analysis of another experiment: Affective blink modulation was obtained even when participants made an explicit reaction time response to startle probes presented during picture viewing (see abstract: Bradley, Drobes, \& Lang, 1996). These findings are important and lift constraints on 
the type of designs that can be used to measure affective modulation of the startle reflex. That is, they show that probe startle modulation is highly robust and clearly obtained even when probes are embedded in a second task.

Replicating earlier findings (Schupp et al., 1997), P300 responses to a secondary acoustic probe were smaller for emotionally arousing pictures (both pleasant and unpleasant) as opposed to neutral low-arousal materials. Furthermore, this pattern of modulation was shown for both startling and nonstartling probe stimuli, and the modulatory effect was again similar regardless of whether the probe was attended or unattended. Using very different foreground tasks, previous studies have found similar patterns of P300 inhibition for attended and unattended secondary probes that varied in their startling characteristics (e.g., Polich, 1989; Roth, Dorato, \& Kopell, 1984). These data are consistent with the hypothesis that reduction of secondary probe P300 when processing affectively intense pictures reflects a greater allocation of attentional resources to these visual stimuli.

A new finding was the modulation of the N100 component by picture valence, an effect that depended on both probe type and task. The N100 was larger for unpleasant than for pleasant pictures, but only for startle probes and only when these probes were attended. This pattern of modulation was reliable and could not be ascribed to blink artifact. It does, however, mimic the blink reflex, with a parallel potentiation of this sensory component of the ERP. This pattern suggests the possibility that defensive motivation may uniquely prime early cortical processing of priority sensory input by acting to enhance such stimuli and producing an ERP response similar to that prompted by an increase in stimulus intensity.

The data of this experiment extend earlier findings indicating that two concurrent responses to a startle probe-the blink reflex and the P300 component of the ERP_-vary differentially with the affective dimensions of pleasure and arousal. According to a motivational analysis of emotion, these dimensions are fundamental in organizing affective response and indicate the behavioral direction and intensity prompted by an affective cue. It is hypothesized that startle blink modulation indexes the extent to which a stimulus engages defensive or appetitive motivational systems in the subcortex of the brain (Lang et al., 1990; Lang, Bradley, \& Cuthbert, 1997). P300 modulation, however, although silent with regard to affective direction, reflects the general motivational pertinence of cues (appetitive or aversive). One interpretation of these findings is that, in natural environments, events that are important for survival automatically recruit natural selective attention processes (Lang et al., 1997). The fact that neither the pleasure-blink nor the arousalP300 relationship were diminished by attention to the startle probe strengthens this view.

\section{REFERENCES}

Anthony, B. J., \& Graham, F. K. (1985). Blink reflex modification by selective attention: Evidence for the modulation of "automatic" processing. Biological Psychology, 20, 43-59.

Bradley, M. M., Drobes, D., \& Lang, P. J. (1996). A probe for all reasons: Reflex and RT measures in perception [Abstract]. Psychophysiology, 33(Suppl.), S25.

Center for the Study of Emotion and Attention [CSEA-NIMH] (1995). The international affective picture system [IAPS; photographic slides]. Gainesville, FL: University of Florida.

Cuthbert, B. N., Schupp, H. T., Bradley, M. M., McManis, M., \& Lang, P. J. (1996). Affective picture viewing: Task and stimulus effects on startle P300 and blink [Abstract]. Psychophysiology, 33(Suppl.), S32.

Graham, F. K. (1992). Attention: The heartbeat, the blink, and the brain. In B. A. Campbell, H. Hayne, \& R. Richardson (Eds.), Attention and information processing in infants and adults (pp. 3-29). Hillsdale, NJ: Erlbaum.

Hackley, S. A., \& Graham, F. K. (1987). Effects of attending to the spatial position of reflex-eliciting and reflex-modulating stimuli. Journal of Experimental Psychology: Human Perception and Performance, 13, 411-424.

Lang, P. J., Bradley, M. M., \& Cuthbert, B. N. (1990). Emotion, attention, and the startle reflex. Psychological Review, 97, 377-395.

Lang, P., Bradley, M. M., \& Cuthbert, B. N. (1997). Motivated attention: Affect, activation, and action. In P. Lang, R. F. Simons, \& M. Balaban
(Eds.), Attention and orienting: Sensory and motivational processes (pp. 97-136). Hillsdale, NJ: Erlbaum.

Miller, G. A., Gratton, G., \& Yee, C. (1988). Generalized implementation of an eye movement correction procedure. Psychophysiology, 25, 241243.

Näätänen, R., \& Picton, T. (1987). The N100 wave of the electric and magnetic response to sound: A review and an analysis of the component structure. Psychophysiology, 24, 375-425.

Polich, J. (1989). P300 from a passive auditory paradigm. Journal of Electroencephalography and Clinical Neurophysiology, 74, 312-320.

Roth, W. T., Dorato, K. H., \& Kopell, B. S. (1984). Intensity and task effects on evoked physiological response to noise bursts. Psychophysiology, 21, 466-481.

Schupp, H. T., Cuthbert, B. N., Bradley, M. M., Birbaumer, N., \& Lang, P. J. (1997). Probe P300 and blinks: Two measures of affective startle modulation. Psychophysiology, 34, 1-6.

(Received March 31, 1997; ACCEPTED August 27, 1997) 